\title{
Pressure Sensitivity of Side-Hole Optical Fiber Sensors
}

\author{
J.R.Clowes $^{(a)}$, S.Syngellakis ${ }^{(b)}$, M.N.Zervas ${ }^{(a)}$ \\ (a)Optoelectronics Research Centre, University of Southampton, \\ Southampton, Hampshire, UK, SO17 1BJ. \\ Tel:+44 1703593954 ; Fax: +44 1703593149 \\ E-Mail: jc@orc.soton.ac.uk
}

(b) Department of Mechanical Engineering, University of Southampton, Southampton, Hampshire, UK.

Tel: +441703592844

\begin{abstract}
:
The dependency of the pressure-induced birefringence of a side-hole fiber on its geometry has been numerically investigated using the finite element method. It is shown that the pressure sensitivity of such a fiber is linearly dependent on $\varphi^{2}$, where $\varphi$ is the angle between the side hole center and core center axis and the core center to side-hole tangent . Experimental data obtained with two different side-hole fiber sensors are shown to agree extremely well (to within $1 \%$ ) with theoretical predictions.
\end{abstract}


John Clowes.

Optoelectronics Research Centre,

University of Southampton,

Southampton,

Hampshire.

SO17 1BJ

UK.

tel: +441703593954

fax: +441703593149

e-mail: jc@orc.soton.ac.uk

18th December 1997.

To whom ever it may concern.

I enclose four copies of our manuscript entitled: "Pressure Sensitivity of Side-Hole Optical Fiber Sensors", for publication in Photonics Technology Letters. I also enclose a copy of the text on 3.5 " disc in microsoft word for windows format.

I hope that the enclosed information is suitable and that this paper will be considered for publication. Please refer to myself as the author for correspondence at the above address.

Yours Sincerely

John Clowes. 


\section{Pressure Sensitivity of Side-Hole Optical Fiber Sensors}

$$
\text { J.R.Clowes }{ }^{(a)} \text {, S.Syngellakis }{ }^{(b)}, \text { M.N.Zervas }{ }^{(a)}
$$

(a) Optoelectronics Research Centre, University of Southampton, Southampton, Hampshire, UK, SO17 1BJ.

$$
\text { Tel:+44 } 1703593954 \text {; Fax: +44 } 1703593149
$$

E-Mail: jc@orc.soton.ac.uk

(b) Department of Mechanical Engineering, University of Southampton, Southampton, Hampshire, UK.

Tel: +44 1703592844

\section{Introduction}

Side-hole fiber was proposed by Xie et al [1] as a possible sensor for hydrostatic or acoustic pressure. In the presence of hydrostatic pressure acting on the fiber surface, an anisotropic stress is induced in the core due to the fiber geometry. Through the photoelastic effect, the result is a pressure-induced birefringence which is a direct measure of the applied pressure.

In this paper we will present experimental data for pressure sensors fabricated from two different geometries of side-hole fiber. We monitor the pressure-induced birefringence in the two sensors and calculate the pressure sensitivity of various fiber geometries. The measurements are performed for limited time and at low temperature to avoid the recently observed sensor drift [2]. 
Using the finite element method (FEM), the two geometries of side-hole fiber are modeled and a comparison is drawn between theory and experiment to show excellent correlation in the pressure sensitivities.

Finally, using the developed FEM model, we investigate the dependence of the pressure sensitivity of side-hole fiber upon the fiber geometry. The model predicts a simple relationship between the fiber sensitivity and the size and position of the side-holes within the structure. This relationship will enable optimization of the pressure sensitivity of such fiber sensors.

\section{Polarimetric Fiber Pressure Sensor}

Figure 1 shows schematically the experimental setup used to measure the birefringence of the side-hole fibers. The sensor head consists of an input fiber polariser aligned at $45^{\circ}$ to the birefringent axes of a length of side-hole fiber (typically between $0.15 \mathrm{~m}$ and $0.3 \mathrm{~m}$ in length). A mirror -ended fiber is spliced to the end of the sensor to increase the reflected power.

The pressure-induced birefringence in the sensing head is remotely monitored using a matched low-coherence interferometer in which the path difference is matched in a reference $\mathrm{Hi}-\mathrm{Bi}$ fiber scanned in temperature [3]. The number of fringes between the central and side fringes in the resulting fringe pattern (Fig.1) is a direct measure of pressure-induced retardation. Knowledge of the length of the side-hole fiber allows a very simple calculation of the pressure-induced birefringence in radians per metre.

All measurements were made at $1300 \mathrm{~nm}$ and hydrostatic pressure was applied to the fiber by means of a dead-weight tester through a liquid medium. 


\section{Experimental Results}

Figure 2 shows photographs of two different side-hole fiber geometries that have been used in pressure sensor measurements. The dimensions of the structure are given in Table 1 where $r_{\mathrm{SH}}$ is the side hole radius, $a$ is the distance between the core and side-hole centers and $\varphi$ is the angle between the side-hole center and core center axis and the core center to side hole tangent (see the inset of Fig. 4). The fibers were fabricated to be 125

- $\mu \mathrm{m}$ in diameter although it is clear that there has been considerable distortion and hole collapse in Fiber B during the fiber pulling procedure. Fiber A clearly has a better circularity than fiber B although there is a small amount of core ellipticity in this example as well.

Figure 3 shows the measured birefringence of fibers $A$ and $B$ with applied pressure. Note that any intrinsic (zero pressure) fiber birefringence due to core ellipticity and fiber residual stresses has been ignored and zero pressure therefore produces zero birefringence.

The relationship for both fibers is a linear one with pressure although the two fiber sensitivities (given in Table I) differ considerably. It can be easily noted that the fiber with large side-holes is the more sensitive of the two by a factor of approximately 6 .

\section{Theory: Finite Element Model}

FEM can be used to predict the localized pressure-induced stresses $\left(\sigma_{x, y, z}\right)$ in the side-hole fiber from which the localized refractive index perturbations $\left(\Delta \mathrm{n}_{\mathrm{x}}\right.$, and $\left.\Delta \mathrm{n}_{\mathrm{y}}\right)$ may be calculated by the photoelastic equations [4]. Assuming that the stress-induced perturbation of the refractive index is small $\left(\Delta \mathrm{n}_{\mathrm{x}, \mathrm{y}} \ll \mathrm{n}_{0}\right)$, an expression may be derived to describe the modal distribution within a perturbed fiber in terms of known modes in an 
unperturbed weakly guiding fiber [5]. With an invariant longitudinal perturbation, the change in propagation constant may be described by:

$$
\beta_{x, y}=\bar{\beta}+\frac{\left[\iint_{A_{x}}\left(n_{x, y}-n_{0}\right) E^{2} d A\right]}{\int_{A_{\infty}} E^{2} d A}
$$

Here, $\beta_{x, y}$ are the perturbed propagation constants in the $\mathrm{x}$ - and $\mathrm{y}$-polarisation directions and $\bar{\beta}$ is the unperturbed propagation constants. $n_{x, y}$ are the perturbed refractive indices in the $\mathrm{x}$ - and $\mathrm{y}$-polarisation directions and $n_{0}$ is the unperturbed refractive index of the material. $k=2 \pi / \lambda$, where $\lambda$ is the free space wavelength. $A$ is the area of the fiber cross section and $\mathrm{E}$ describes the electric field distribution. The fiber birefringence can therefore be calculated simply using the expression $\Delta \beta=\beta_{x}-\beta_{y} \quad$ (in $\mathrm{rad} / \mathrm{m}$ ).

The fiber has been modeled as closely as possible to a true side-hole fiber manufactured from a standard telecommunications grade preform; fiber radius $r_{f}=62.5 \mu \mathrm{m}$, core radius $r_{c}=4 \mu \mathrm{m}$, cladding Young modulus $\dot{E}_{c l}=72 \mathrm{GPa}$ and Poisson ratio $v_{c l}=0.173$, the $3 \%$ germanium doped core Young modulus $E_{c}=70.8 \mathrm{GPa}$ and Poisson ratio $v_{c}=0.165$ [6].

The location and size of the side-holes within the fiber are determined by the side-hole radius $\left(\mathrm{r}_{\mathrm{SH}}\right)$ and the core center to side-hole center separation (a) as shown in the inset of Figure 4.

A two-dimensional stress analysis was performed using ANSYS, a general-purpose FEM 
package [7]. The FEM output includes the centroid co-ordinates, the area and the $\mathrm{x}$ and $\mathrm{y}$ normal stresses for every element. Calculation of the birefringence is therefore achieved by calculating $\Delta \mathrm{n}_{\mathrm{x}, \mathrm{y}}$ for each element using the photoelastic equations, calculating the respective field magnitude for each element and finally evaluating $\beta_{x}$ and $\beta_{y}$ from equation (1).

The model uses parameters for a standard telecommunication-grade fiber with a numerical aperture of 0.12 , core index of 1.462 , cladding index of 1.457 and a core radius of $4 \mu \mathrm{m}$. The operating wavelength is taken as $1300 \mathrm{~nm}$ as this wavelength was used in the laboratory pressure measurements.

\section{Numerical Results}

The solid lines of Figure 3 show the modeled birefringence versus applied pressure (in psi,1psi $6895 \mathrm{~Pa}$ ) for fiber geometries $\mathrm{A}$ and $\mathrm{B}$. This result shows excellent agreement between the model and experiment for fiber A ( to approximately $99 \%$ accuracy). For fiber $B$, an accuracy of approximately $91 \%$ is achieved. Here, the larger discrepancy can be attributed to the non-circular fiber, hole and core shapes which are consequences of side-hole collapse during fiber fabrication.

A simple observation of the results of Figure 3 shows that the fiber with large holes (fiber A) has a much greater sensitivity to pressure (approximately 6 times) than a fiber with small holes separated a greater distance from the core (fiber B). The model has therefore been utilized to investigate the relationship between the side-hole position and size and the pressure sensitivity of the fiber. 
Side-hole fibers with the same hole position $\left(a-r_{S H}\right)$ but varying hole radius $\left(r_{S H}\right)$ have been modeled using values for the core to side-hole separation $\left(a-r_{S H}\right)$ of 6,12 and $18 \mu \mathrm{m}$. By varying the side-hole radius and location, the angle $\varphi$ (see inset of Fig.4) is effectively being varied.

Figure 4 shows the results for the pressure induced birefringence of the three cases mentioned when plotted against $\varphi^{2}$. The curves all show that the pressure sensitivity of the fiber follows a highly linear relationship with $\varphi^{2}$ for values of $\varphi$ up to approximately $45^{\circ}$. Examination of the fibers $A$ and $B$ and their respective values of $\varphi$ suggests that fiber A should have approximately 5.3 times the pressure sensitivity as fiber B. The actual measured value is about 6 times. However, the FEM analysis has considered fiber cross sections of perfect cladding and core circularity which is obviously not true for fiber $\mathrm{B}$ and to a lesser extent, fiber $\mathrm{A}$. The agreement between the model and the experimental data and also the $\varphi^{2}$ relationship is therefore considered to be very good.

Figure 4 also shows that the sensitivity of the fiber is not significantly affected by the positioning of the holes as all three lines have a very similar gradient. This is an extremely interesting and useful result in terms of the manufacturing of such fibers. It suggests that a fiber with small holes near to the core will have a high pressure sensitivity. This will make it possible to fabricate a highly pressure sensitive side-hole fiber whilst maintaining a relatively strong structure.

For angles greater than $45^{\circ}$, the sensitivity tended to deviate from the straight line law. This has been attributed to difficulties with the meshing process of the finite elements as 
the side-holes become very large and form the majority of the cross sectional area. In reality this is not a problem as such a fiber geometry would be extremely fragile and therefore impractical.

\section{Conclusions}

We have modeled the pressure sensitivity of side-hole fiber sensors using the method of finite elements and have shown excellent agreement between the model results and sidehole fibers used in laboratory high pressure experiments. By investigating the effect of geometry on pressure sensitivity, we have shown a linear relationship with $\varphi^{2}$, where $\varphi$ is the angle between the side-hole center and core center axis and the core center to sidehole tangent. Two fibers used in laboratory pressure measurements with values of $\varphi$ of $42.39^{\circ}$ and $18.46^{\circ}$, show a very good agreement with this $\varphi^{2}$ relationship. Any discrepancy (of approximately $10 \%$ in the worst case) is attributed to core and fiber noncircularities . Finally, we predict that a highly pressure-sensitive fiber may be fabricated with small holes located close to the core to maintain fiber strength and integrity .

We would like to acknowledge Sensor Dynamics and Chevron for funding of this work. Helpful discussions with Prof.D.N.Payne, Dr.E.Kluth and Dr.M.P.Varnham are gratefully acknowledged. 


\section{References}

[1] H.M.Xie, Ph. Dabkiewicz, R. Ulrich, K. Okamoto, "Side-hole fiber for fiber-optic pressure sensing”, Optics Letters, 1986, Vol.11, No.5, pp.333-335

[2] J.R.Clowes, J. McInnes, M.N.Zervas, D.N.Payne, "Effects of high temperature and pressure on silica optical fibers and the implications on optical fiber sensors", presented at the Optical Fiber Sensors conf., 1997, paper OFB2.

[3] M.G.Xu, M.Johnson, M.Farhadiroushan, J.P.Dakin, "Novel polarimetric fiber device for interrogating white-light interferometers", Electronics Letters, 1993, V.29, No.4, pp.378-379.

[4] A.Yariv, P.Yeh, "Optical Waves in Crystals”, J.Whiley \& Sons, N.York, (1984), Chap 9.

[5] A.W.Snyder, J.D.Love, "Optical waveguide Theory", Chapman \& Hall, London, 1983, pp.375-376.

[6] N.P.Bansal, "Handbook of Glass Properties", Academic Press Inc, London, 1986.

[7] ANSYS User's Manual for Revision 5.1 - Volumes I-IV, Swanson Analysis Systems, Housten, Pennsylvania, 1994. 


\section{Figure Captions:}

Figure l: Schematic of the experimental setup used to measure th pressure-induced birefringence in side-hole fiber and a typical output fringe pattern.

Figure 2: Cross-section photographs of (a) Fiber A and (b) Fiber B.

Figure 3: Predicted and measured pressure-induced birefringence of Fiber A and Fiber B.

Figure 4: Predicted pressure-induced birefringence $V s \varphi^{2}$ for three fibers with core to side-hole separations of 6,12 and $18 \mu \mathrm{m}$. (Inset: Schematic of the fiber geometry).

Table 1: Summary of fiber geometry parameters and sensitivity results. 


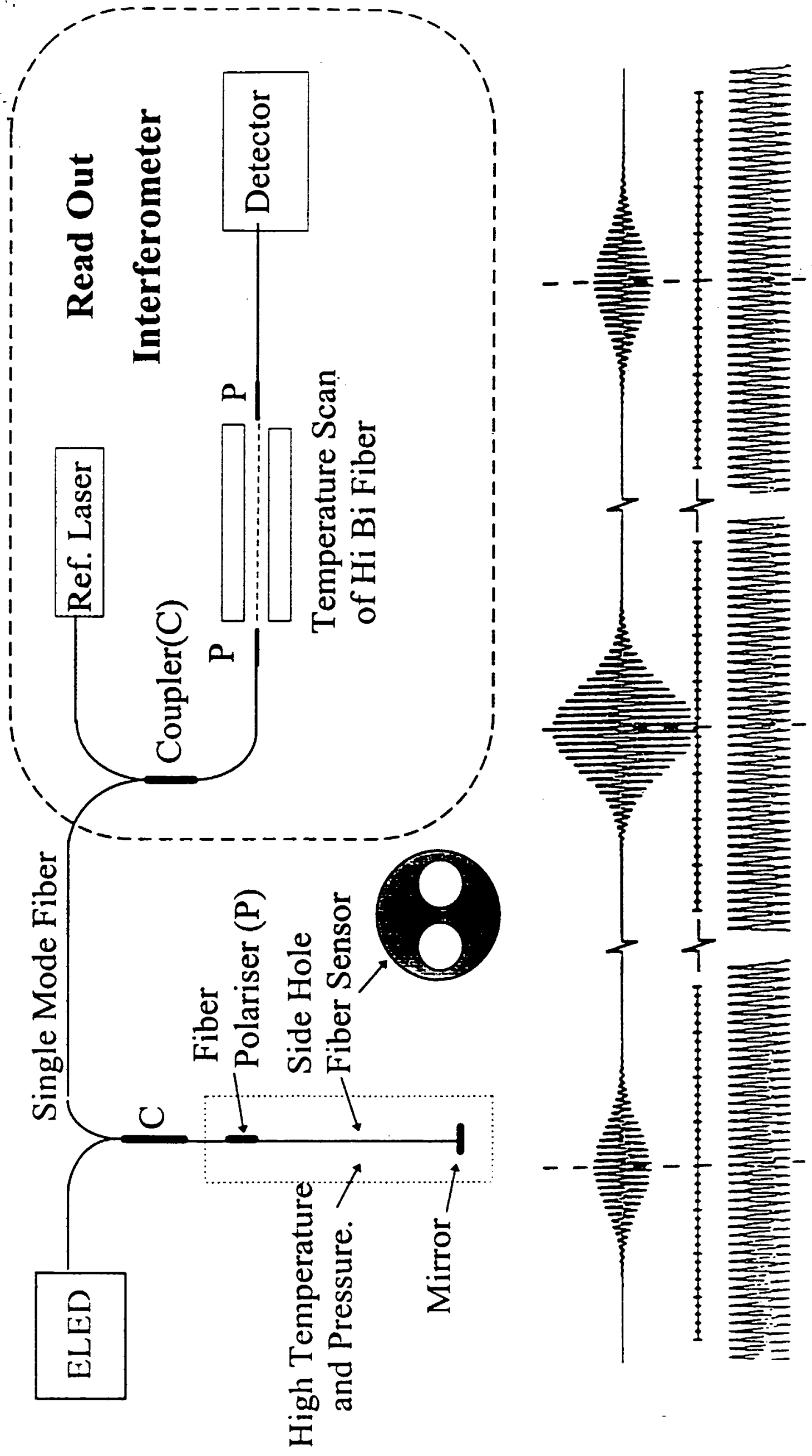



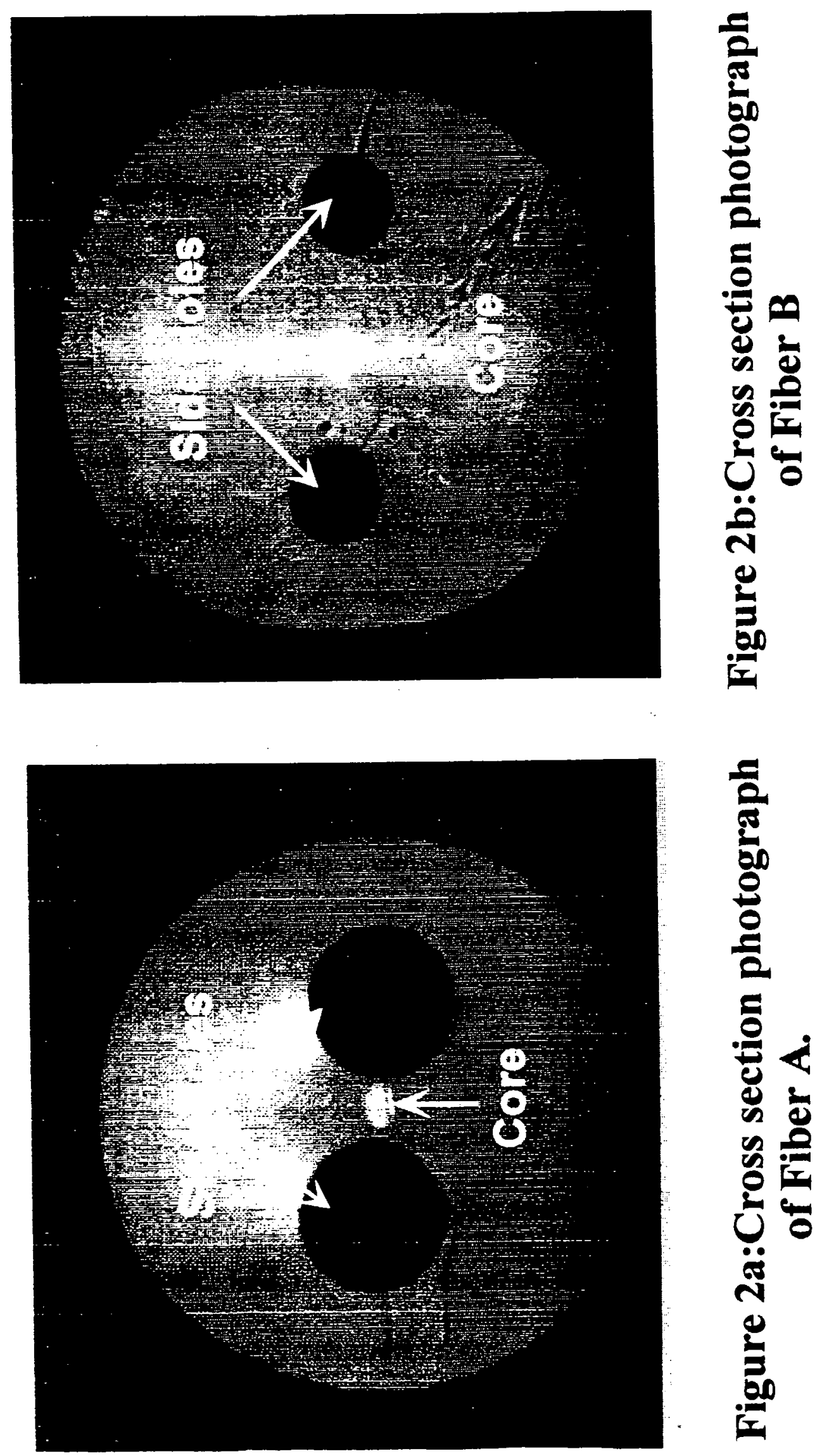


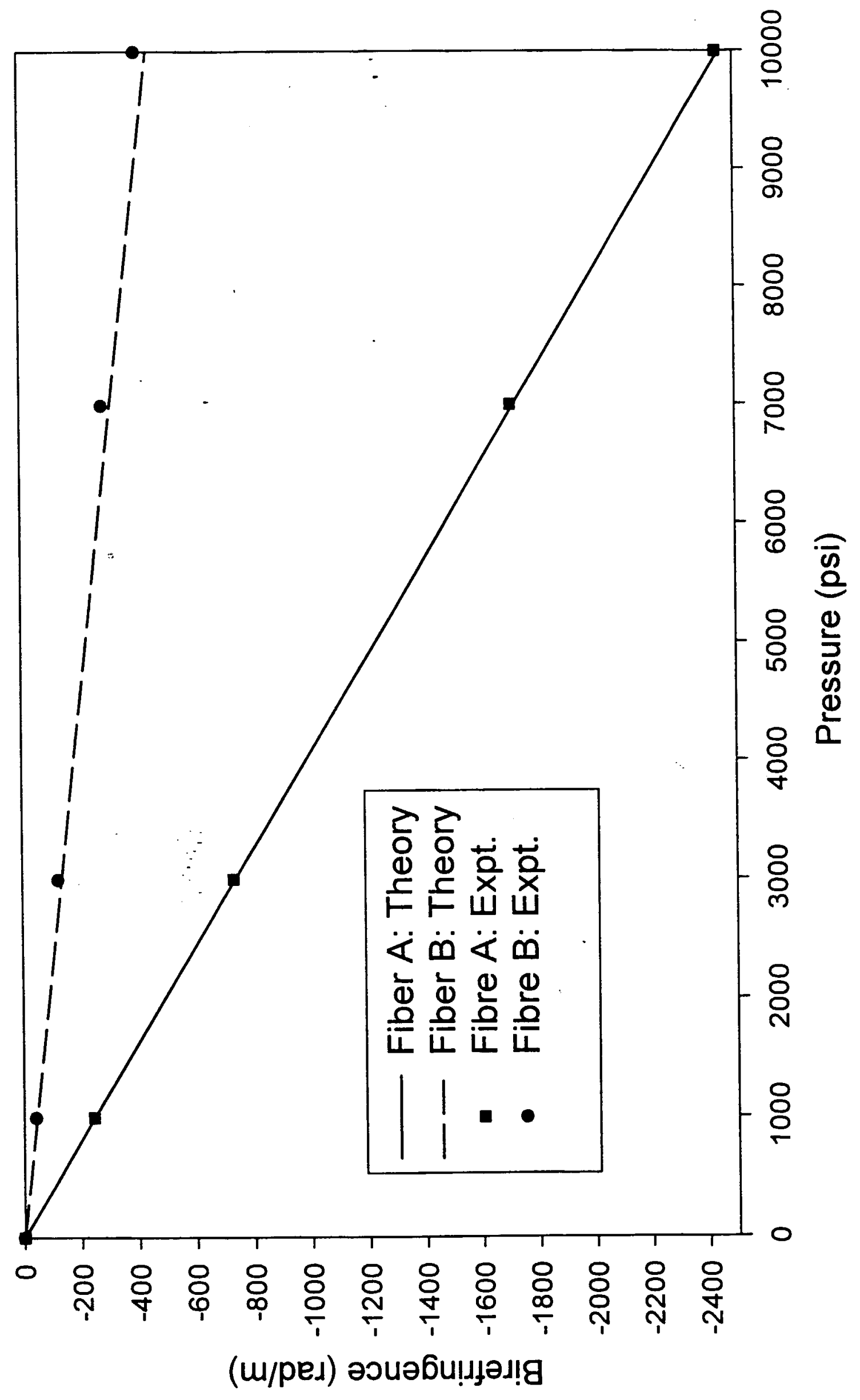




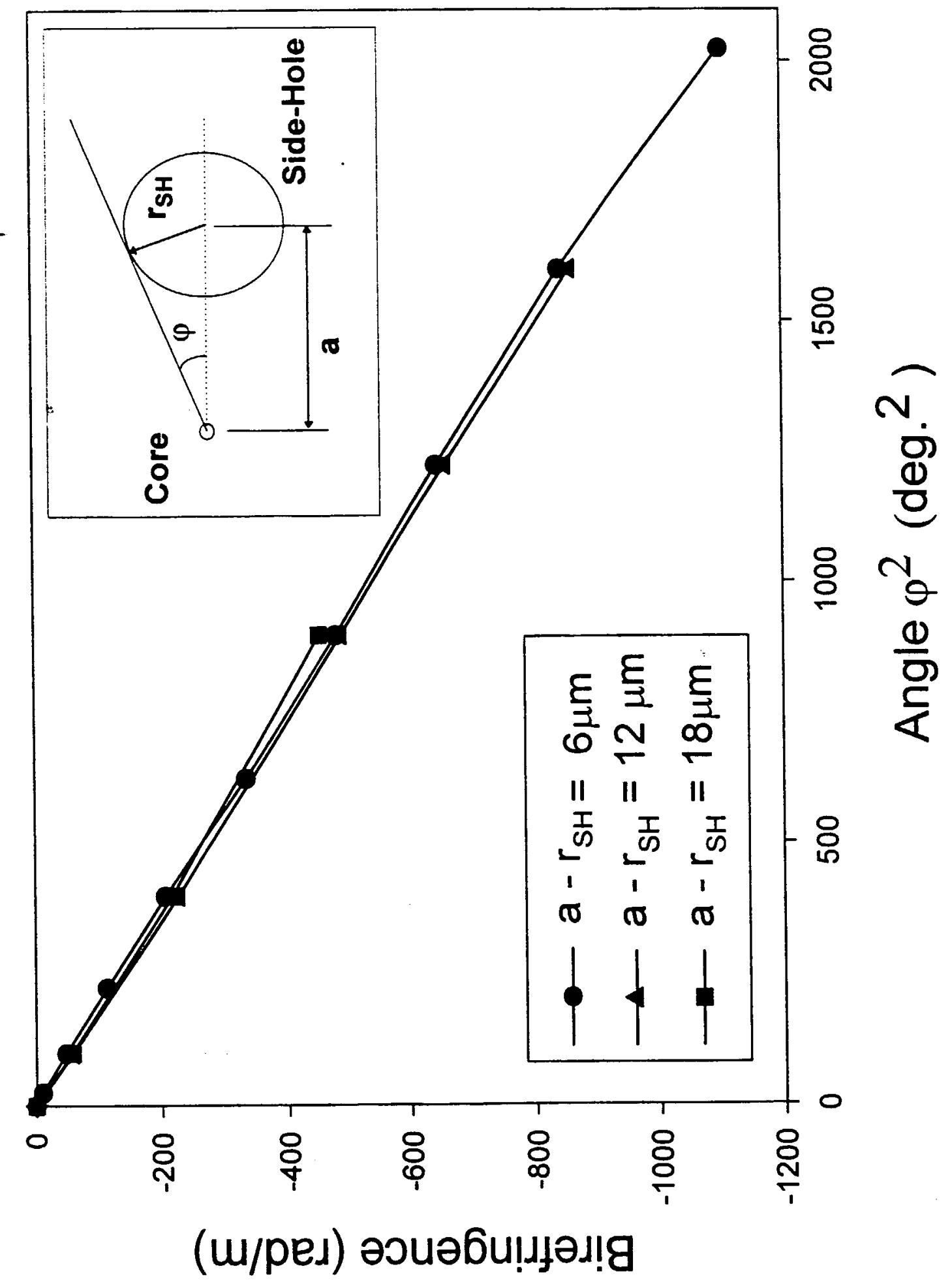




\begin{tabular}{|l|c|c|c|c|}
\hline Fiber & $\mathrm{a}(\mu \mathrm{m})$ & $\mathrm{r}_{\mathrm{SH}}(\mu \mathrm{m})$ & $\varphi($ deg. $)$ & $\begin{array}{c}\text { Sensitivity } \\
(\mathrm{mrad} / \mathrm{m} \mathrm{psi})\end{array}$ \\
\hline $\mathrm{A}$ (Theory) & 24.46 & 16.49 & 42.39 & 246.07 \\
\hline $\mathrm{B}$ (Theory) & 9.50 & 30.0 & 18.46 & 44.88 \\
\hline A (Expt.) & $\approx 24.46$ & $\approx 16.49$ & $\approx 42.39$ & 244.13 \\
\hline B (Expt.) & $\approx 9.50$ & $\approx 30.0$ & $\approx 18.46$ & 40.75 \\
\hline
\end{tabular}

